Hardy-Ramanujan Journal

Vol.20 (1997) 29-39

\title{
ON THE ZEROS OF A CLASS OF GENERALISED DIRICHLET SERIES-XIX
}

\section{BY}

\section{K. RAMACHANDRA}

\section{§ 1. INTRODUCTION AND PRELIMINARIES. The object of this} paper is to give a simpler approach to the following theorem (this theorem namely Theorem 10 of XVIII ${ }^{[5]}$ constitutes the main theorem on BalasubramanianRamachandra functions arising out of the works III ${ }^{[1]}, \mathrm{IV}^{[2]}, \mathrm{V}^{[6]}, \mathrm{VI}^{[3]}$ due to R. Balasubramanian and K. Ramachandra).

THEOREM 1. (R. BALASUBRAMANIAN AND K. RAMACHANDRA) (i) Let $\lambda_{n}(n=1,2,3, \cdots)$ be an increasing sequence of positive real numbers such that $\lambda_{n+1}-\lambda_{n}$ is bounded both above and below. This sequence will be further restricted by the condition (vii) or (viii) as the case may be. $\theta$ will denote a real constant.

Let $f(x)$ and $g(x)$ be positive real valued functions defined in $x \geq 0$, satisfying

(ii) $f(x) x^{\eta}$ is monotonic increasing and $f(x) x^{-\eta}$ is monotonic decreasing for every fixed $\eta>0$ and all $x \geq x_{0}(\eta)$.

(iii) $\lim _{x \rightarrow \infty}\left(g(x) x^{-1}\right)=1$.

(iv) $g(x)$ is differentiable once for $x \geq 0$ and $g^{\prime}(x)$ lies between two positive constants. Also $g(x)$ is tuice differentiable for $x \geq x_{0}$ and $\left(g^{\prime}(x)\right)^{2}-$ $g(x) g^{\prime \prime}(x)$ lies between two positive consiants for $x \geq \vec{x}_{0}$. 
Let $\left\{a_{n}\right\}$ and $\left\{b_{n}\right\}(n=1,2,3, \cdots)$ be two sequences of complex numbers having the following properties

(i) ${ }_{1} \dot{b}_{n} !(f(n))^{-1}$ lies between two positive constants (for all integers $n \geq n_{0}$ ) and $\left(\sum_{n \leq x}\left|a_{n}\right|^{2}\right) x^{-1}$ does not exceed a positive constant for all $x \geq 1$.

(vi) For all $x \geq 1, \sum_{x \leq n \leq 2 x}\left|b_{n+1}-v_{n}\right| \ll f(x)$.

We next assume that $\left\{a_{n}\right\}$ and $\left\{b_{n}\right\}$ satisfy at least one of the following conditions. We set $\lambda_{n}=g(n)$.

(vii) MONOTONICITY CONDITION. There exists an arithmetic progression. 4 (of natural numbers) such that

$$
\lim _{x \rightarrow \infty}\left(x^{-1} \sum_{n \leq x}^{\prime} a_{n}\right)=h(0<|h|<\infty),
$$

where the accent denotes the restriction of $n$ to $\mathcal{A}$. Also for every positive constant $\nu$ we have $\left|b_{\eta}\right| \lambda_{n}^{-\nu}$ is monotonic decreasing for all $n\left(\geq n_{0}\right)$ in $\mathcal{A}$.

(viii) REAL PART CONDITION. There exists an arithmetic progression $\mathcal{A}$ (of natural numbers) such that

$$
\lim \inf _{x \rightarrow \infty}\left(\frac{1}{x} \sum_{x \leq \lambda_{n} \leq 2 x, R e}^{\prime} \operatorname{Re} a_{n}>0\right.
$$

and

$$
\lim _{x \rightarrow \infty}\left(x^{-1} \sum_{x \leq \lambda_{n} \leq 2 x, \operatorname{Re} a_{n}<0}^{\prime} \text { Re } a_{n}\right)=0
$$

where the accent denotes the restriction of $n$ to $A$.

(ix) Finally let $\left\{\alpha_{n}\right\}(n=1,2,3, \cdots)$ be a sequence of real numbers such that $\left|\alpha_{n}\right|$ does not exceed a small positive constant (depending on other constants). We suppose that the series

$$
F(s)=\sum_{n=1}^{\infty} a_{n} b_{n} e^{2 \pi i n \theta}\left(\lambda_{n}+\alpha_{n}\right)^{-s} \quad(\text { Re } s \geq 2)
$$


can be continued analytically in $\left(\sigma \geq \frac{1}{2}-\delta, T-\log T \leq t \leq 2 T+\log T\right)$ (where $\delta$ is a positive constant) and there $\log \max (|F(s)|+100) \ll \log T$. As usual we have written $s=\sigma+i t$.

Then on every line segment $\left(\sigma=\frac{1}{2}-\delta_{4}, T \leq t \leq 2 T\right)$, ( $\delta_{4}$ being any constant with $\left.0<\delta_{4}<\delta\right)$ there are $\gg T(\log \log T)^{-1}$ well-spaced Titchmarsh points with the lower bound $\gg T^{\delta_{4}} f(T)$ for $|F(s)|$. If further

$$
\frac{1}{T} \int_{T-\sqrt{\log T}}^{2 T+\sqrt{\log T}}\left|F\left(\frac{1}{2}-\delta_{4}+i t\right)\right|^{2} d t \ll T^{2 \delta_{4}}(f(T))^{2}
$$

for every constant $\delta_{4}$ (with $\left.0<\delta_{4} \leq \delta\right)$, then there are $\gg T$ well-spaced Titchmarsh points on every line segment $\left(\sigma=\frac{1}{2}-\delta_{4}, T \leq t \leq 2 T\right)$ with the lower bound $\gg T^{\delta_{4}} f(T)$ for $|F(s)|$.

In other words there exist real numbers $t_{1}, t_{2}, \cdots, t_{r}\left(\right.$ with $r \gg T(\log \log T)^{-1}$ and $r \gg T$ respectively in the two cases) such that $T \leq t_{j} \leq 2 T(j=$ $1,2, \cdots, r)$, the minimum of $\left|t_{j}-t_{j^{\prime}}\right|$ taken over all pairs $\left(j, j^{\prime}\right)$ with $j \neq j^{\prime}$ is bounded below and further

$$
\left|F\left(\frac{1}{2}-\delta_{4}+i t_{j}\right)\right| \gg T^{\delta_{4}} f(T) .
$$

The proof of this theorem depends on the following two lemmas.

LEMMA 1 (van-der-CORPUT). If $f_{1}(x)$ is real and twice differentiable and $0<\mu_{2} \leq f_{1}^{\prime \prime}(x) \leq h^{\prime} \mu_{2}$ (or $\left.\mu_{2} \leq-f_{1}^{\prime \prime}(x) \leq h^{\prime} \mu_{2}\right)$ throughout the interval $[a, b]$, and $b \geq a+1$, then

$$
\sum_{a<n \leq b} \operatorname{Exp}\left(2 \pi i f_{1}(n)\right)=O\left(h^{\prime}(b-a) \mu_{2}^{\frac{1}{2}}\right)+O\left(\mu_{2}^{-\frac{1}{2}}\right) .
$$

REMARK. This result is Theorem 5.9 on page 104 of [8], with a slight change of notation.

LEMMA 2 (H.L. MONTGOMERY AND R.C. VAUGHAN). If $\left\{\lambda_{n}\right\}$ is any incredsing sequence of real numbers and $\left\{A_{n}\right\}$ and $\left\{B_{n}\right\}$ are any two sequences of complex numbers, then

$$
\left|\sum \sum_{m \neq n} \frac{A_{m} \bar{B}_{n}}{\log \left(\lambda_{m} \lambda_{n}^{-1}\right)}\right| \leq K\left(\sum \delta_{n}^{-1}\left|A_{n}\right|^{2}\right)^{\frac{1}{2}}\left(\sum \delta_{n}^{-1}\left|B_{n}\right|^{2}\right)^{\frac{1}{2}}
$$


where $\delta_{n}=\min _{m \neq n}\left|\lambda_{n}-\lambda_{m}\right|$ and $K$ is a numerical constant.

REMARK. We need only a special case of this result where $\lambda_{n+1}-\lambda_{n}$ lies between two positive constants (and so the same is true of $n \delta_{n}$ ). For the proof in this special case and also for a reference to the paper of Montgomery and Vaughan see [7].

\section{§ 2. SOME MORE LEMMAS.}

LEMMA 3. Let $y>0, w=u+i v, R(w)=\operatorname{Exp}\left(\left(\operatorname{Sin} \frac{w}{100}\right)^{2}\right)$, and

$$
\Delta(y)=\frac{1}{2 \pi i} \int_{u=2} y^{w} R(w) \frac{d w}{w} .
$$

Then for $|u| \leq 3$ we have $|R(w)| \ll\left(E x p \operatorname{Exp}\left|\frac{v}{100}\right|\right)^{-1}$. Consequently $\Delta(y)=1+O\left(y^{-2}\right)$ and also $\Delta(y)=O\left(y^{2}\right)$.

PROOF. By trivial computation (and moving the line of integration to $u=-2$ and $u=2$ respectively).

$\mathcal{A}$ will denote the arithmetic progression consisting of an infinite subset of natural numbers. Let $\lambda(0<\lambda<1)$ be a constant. We put $X=T \lambda$ (later we will choose $\lambda$ to be a small constant). $S$ will denote the set $\mathcal{A} \cap\left[\frac{1}{2} X, X\right]$. All our $O$-constants and the constants implied by the Vinogradov symbols $\gg$ and $\ll$ will be independent of $\lambda$.

LEMMA 4. For $T \leq t \leq 2 T$, we have,

$$
\left|\sum_{n \in S} E x p(-2 \pi i n \theta+i t \log g(n))\right| \ll T^{\frac{1}{2}} .
$$

PROOF. Noting that the second derivative of $-2 \pi x \theta+t \log g(x)$ is $t\left(\left(g^{\prime}(x)\right)^{2}-g(x) g^{\prime \prime}(x)\right)(g(x))^{-2}$ the lemma follows by Lemma 1.

LEMMA 5. For $T \leq t \leq 2 T$, we have

$$
\left|\sum_{n \in S} \bar{b}_{n} \operatorname{Exp}(-2 \pi i n \theta+i t \log g(n))\right| \ll T^{\frac{1}{2}} f(X) \text {. }
$$

PROOF. The proof follows by partial summation (from Lemma 4) on using $\sum_{x \leq n \leq 2 x}\left|b_{n+1}-b_{n}\right| \ll f(x)$ for all $x \geq 1$. 
Next we put. $g(n)=\lambda_{n}$. We consider the case $\alpha_{n} \equiv 0$ first. Our object is to obtain a good lower bound for the LHS of (2) below.

IEMMA 6. For $s=\frac{1}{2}-\delta+i t, T \leq t \leq 2 T$, put

$$
F_{X}(s)=\sum_{n=1}^{\infty} a_{n} b_{n} \operatorname{Exp}(2 \pi i n \theta) \lambda_{n}^{-s} \Delta\left(\frac{X}{\lambda_{n}}\right) .
$$

Then

$$
\frac{1}{T} \int_{T}^{2 T}\left|F_{X}(s)\right| d t \gg\left(T^{\frac{3}{2}} f(X)\right)^{-1}|I|
$$

where

$$
I=\int_{T}^{2 T} F_{X}(s) \sum_{n \in S} \bar{b}_{n} \operatorname{Exp}\left(-2 \pi i n \theta+i t \log \lambda_{n}\right) d t .
$$

PROOF. Follows from Lemma 5.

LEMMA 7. We have,

$$
I=T \sum_{n \in S} a_{n}\left|b_{n}\right|^{2} \lambda_{n}^{-\frac{1}{2}+\delta}+O(J)
$$

where

$$
J=\left(J_{1} J_{2}\right)^{\frac{1}{2}}, J_{1}=\sum_{n=1}^{\infty}\left|a_{n} b_{n}\right|^{2} n^{2 \delta}\left(\Delta\left(\frac{X}{\lambda_{n}}\right)\right)^{2}
$$

and

$$
J_{2}=\sum_{n \in\left[\frac{1}{2} X, X\right]} n\left|b_{n}\right|^{2} .
$$

PROOF. Follows from Lemma 2.

LEMMA 8. We have

$$
J_{1}=O\left(X^{1+2 \delta}(f(X))^{2}\right)
$$

and

$$
J_{2}=O\left(X^{2}(f(X))^{2}\right)
$$

PROOF. Follows from $\Delta(y)=1+O\left(y^{-2}\right)=O\left(y^{2}\right)$ and also from (ii) of Theorem 1. 
LEMMA 8. Let

$$
\sum_{0}=\sum_{n \in S} a_{n}\left|b_{n}\right|^{2} \lambda_{n}^{-\frac{1}{2}+\delta} \Delta\left(\frac{X}{\lambda_{n}}\right)
$$

Then

$$
I=T \sum_{0}+O\left(X^{\frac{3}{2}+\delta}(f(X))^{2}\right)
$$

PROOF. Follows from Lemmas 7 and 8 .

LEMMA 10. Under monotonicity condition, we have,

$$
\left|\sum_{0}\right| \gg|h| X^{\frac{1}{2}+\delta}(f(X))^{2}
$$

PROOF. We write

$$
\frac{1}{x} \sum_{n \in \mathcal{A}, n \leq x} a_{n}=h+\varepsilon_{x}
$$

where $\varepsilon_{x} \rightarrow 0$ as $x \rightarrow \infty$. We obtain the result by the monotonicity of $\left|b_{n}\right|^{2} \lambda_{n}^{-\frac{1}{4}+\frac{1}{2} \delta}$.

LEMMA 11. Under the real part condition, we have,

$$
\operatorname{Re} \sum_{0} \gg X^{\frac{1}{2}+\delta}(f(X))^{2}
$$

PROOF. Follows since the contribution from those $a_{n}$ with $R e a_{n}<0$ is of a smaller order.

LEMMA 12. We have

$$
|I|>C_{1} T(f(X))^{2} X^{\frac{1}{2}+\delta}-C_{2}(f(X))^{2} X^{\frac{3}{2}+\delta}
$$

where $C_{1}$ and $C_{2}$ are positive constants independent of $\lambda$.

PROOF. Follows from Lemma 7 to 11.

LEMMA 13. We have, with $s=\frac{1}{2}-\delta+i t, X=T \lambda$, where $\lambda(>0)$ is some fixed small constant, the inequality

$$
\frac{1}{T} \int_{T}^{2 T}\left|F_{X}(s)\right| d t \gg T^{\delta} f(T) .
$$


PROOF. RHS of (13) is

$$
\left(C_{1} T^{\frac{3}{2}+\delta} \lambda^{\frac{1}{2}+\delta}-C_{2} T^{\frac{3}{2}} \lambda^{\frac{3}{2}+\delta}\right)(f(X))^{2} .
$$

Using (ii) it follows that $f(X) X^{-1} \geq f(T) T^{-1}$ and so $f(X) \geq \lambda f(T)$. Lemma 13 follows on fixing $\lambda$ to be a small positive constant.

LEMMA 14. Let now $Y=T \lambda^{\prime}$ where $\lambda^{\prime}\left(0<\lambda^{\prime}<\lambda\right)$ is a small constant. We have

$$
\frac{1}{T} \int_{T}^{2 T}\left|F_{Y}(s)\right| d t \leq \eta_{0} T^{\delta} f(T),
$$

where $\eta_{0}$ depends on $\lambda^{\prime}$ and is small enough if $\lambda^{\prime}$ is small.

PROOF. Note that

$$
\frac{1}{T} \int_{T}^{2 T}\left|F_{Y}(s)\right|^{2} d t \ll Y^{2 \delta}(f(Y))^{2}
$$

and that here RHS is $\leq Y^{\delta}\left(Y^{\frac{1}{2} \delta} f(Y)\right)^{2} \leq\left(\lambda^{\prime}\right)^{\delta} T^{2 \delta}(f(T))^{2}$. Lemma 14 follows from this on using Hölder's inequality.

LEMMA 15. We have, with $X=T \lambda_{1} Y=T \lambda^{\prime}$ where $\lambda$ is as before and $\lambda^{\prime}\left(0<\lambda^{\prime}<\lambda\right)$ is fixed to be a sufficiently small constant, the inequality

$$
\frac{1}{T} \int_{T}^{2 T}\left|F_{X}(s)-F_{Y}(s)\right| d t \gg T^{\delta} f(T)
$$

PROOF. Follows from Lemma 13 and 14.

From now on we fix the positive constants $\lambda$ and $\lambda^{\prime}$ so that (16) is satisfied.

LEMMA 16. Now let $\alpha_{n}$ be real and let $\left|\alpha_{n}\right|$ be bounded above by a small positive constant. Then with $s=\frac{1}{2}-\delta+i t, X=T \lambda, Y=T \lambda^{\prime}$ we have

$$
\begin{gathered}
\frac{1}{T} \int_{T}^{2 T} \mid \sum_{n=1}^{\infty} a_{n} b_{n} \operatorname{Exp}(2 \pi i n \theta)\left(\lambda_{n}+\alpha_{n}\right)^{-s} \\
\left(\Delta\left(\frac{X}{\lambda_{n}+\alpha_{n}}\right)-\Delta\left(\frac{Y}{\lambda_{n}+\alpha_{n}}\right)\right) \mid d t \gg T^{\delta} f(T) .
\end{gathered}
$$


PROOF. We split the infinite serie: on the LHS of (17) to be $\sum_{1}$ with $n \leq T \lambda^{\prime \prime}$ (where $\lambda^{\prime \prime}(>0)$ is a small constant) and $\sum_{2}$ the rest. Clearly (by [,emma 2)

$$
\frac{1}{T} \int_{T}^{2 T}\left|\sum_{1}\right| d t \ll\left(\lambda^{\prime \prime}\right)^{\frac{1}{4} \delta} T^{\delta} f(T)
$$

and also in $\sum_{2}$ using

$$
\begin{gathered}
\left(\lambda_{n}+\alpha_{n}\right)^{-s}\left(\Delta\left(\frac{X}{\lambda_{n}+\alpha_{n}}\right)-\Delta\left(\frac{Y}{\lambda_{n}+\alpha_{n}}\right)\right)-\lambda_{n}^{-s}\left(\Delta\left(\frac{X}{\lambda_{n}}\right)-\Delta\left(\frac{Y}{\lambda_{n}}\right)\right) \\
=\int_{0}^{\alpha_{n}} \frac{d}{d k}\left(\left(\lambda_{n}+k\right)^{-s}\left(\Delta\left(\frac{X}{\lambda_{n}+k}\right)-\Delta\left(\frac{Y}{\lambda_{n}+k}\right)\right)\right) d k
\end{gathered}
$$

and Lemma 2 we are led to Lemma 16. (For details see page 173 of $\mathrm{XIV}^{[4]}$ ).

LEMMA 17. We have, with $\alpha_{n}$ as in Lemma 16,

$$
\begin{gathered}
\frac{1}{T} \int_{T}^{2 T} \mid \sum_{n=1}^{\infty} a_{n} b_{n} \operatorname{Exp}(2 \pi i n \theta)\left(\lambda_{n}+\alpha_{n}\right)^{-s} \\
\left.\left(\Delta\left(\frac{X}{\lambda_{n}+\alpha_{n}}\right)-\Delta\left(\frac{Y}{\lambda_{n}+\alpha_{n}}\right)\right)\right|^{2} d t \ll T^{, 2 \delta}(f(T))^{2} .
\end{gathered}
$$

PROOF. Follows from Lemma 2.

THEOREM 2. Denote by $G(s)$ the infinite series in the LHS of (18). Then there are real numbers $t_{1}, t_{2}, \cdots, t_{r}$ as in Theorem 1 with $r \gg T$ and

$$
\left|G\left(\frac{1}{2}-\delta+i t_{j}\right)\right| \gg T^{\delta} f(T)
$$

PROOF. Divide the interval $[T, 2 T]$ (of integration) on the LHS of (17) into abutting intervals of length 1 , ignoring a bit at one end. Ignore the integrals over intervals of length 1 which do not exceed a small (positive) constant times $T^{\delta} f(T)$. Now apply Hölder's inequality for the rest and apply Lemma 17. We obtain (19).

THEOREM 3. We have

$$
\frac{1}{T} \int_{T-\sqrt{\log T}}^{2 T+\sqrt{\log T}}\left|F\left(\frac{1}{2}-\delta+i t\right)\right| d t \gg T^{\delta} f(T) .
$$


PROOF. follows from Lemma 16 on writing $G(s)$ as a line integral over $u=2$ and moving the line of integration to $u=0$ using siitable horizontal connecting lines.

§ 3. COMPLETION OF THE PROOF OF THEOREM 1. Using the mean square upper bound for $|F(s)|$ and also Theorem 3 , we can obtain (as in the proof of Theorem 2) real numbers $t_{1}, t_{2}, \cdots, t_{r}$ as in Theorem 1 with $r \gg T$ and

$$
\left|F\left(\frac{1}{2}-\delta+i t_{j}\right)\right| \gg T^{\delta} f(T)
$$

Next we use Theorem 2. Out of the numbers $t_{1}, \cdots, t_{r}$ we can omit a minimal number of them and obtain numbers $\tau_{1}, \cdots, \tau_{r^{\prime}}$ such that $r^{\prime} \gg T(\log \log T)^{-1},\left|\tau_{j}-\tau_{j^{\prime}}\right| \gg \log \log T$ for all pairs $\left(j, j^{\prime}\right)$ with $j \neq j^{\prime}$ and

$$
\left|G\left(\frac{1}{2}-\delta+i \tau_{j}\right)\right| \gg T^{\delta} f(T) .
$$

Now writing $G(s)$ as a line integral over $u=2$ and moving the line of integration to $u=0$ using suitable connecting horizontal lines. We thus obtain points $\tau_{1}^{\prime}, \cdots, \tau_{r}^{\prime}$, with

$$
\left|F\left(\frac{1}{2}-\delta+i \tau_{j}^{\prime}\right)\right| \gg T^{\delta} f(T)\left(j=1,2, \cdots, r^{\prime}\right) .
$$

This proves Theorem 1 completely. 


\section{REFERENCES}

[1] R. BALASUBRAMANIAN AND K. RAMACHANDRA, On the zeros of a class of generalised Dirichlet series-III, J. Indian Math. Soc., 41 (1977), 301-315.

[2] R. BALASUBRAMANIAN AND K. RAMACHANDRA, On the zeros of a class of generalised Dirichlet series-IV, J. Indian Math. Soc., 42 (1978), 135-142.

[3] R. BALASUBRAMANIAN AND K. RAMACHANDRA, On the zeros of a class of generalised Dirichlet series-VI, Arkiv för Mathematik, 19 (1981), 239-250.

[4] R. BALASUBRAMANIAN AND K. RAMACHANDRA, On the zeros of a class of generalised Dirichlet series-XIV, Proc. Indian Acad. Sci. (Math. Sci.), 104 (1994), 167-176.

[5] R. BALASUBRAMANIAN, K. RAMACHANDRA AND A. SANKARANARAYANAN, On the zeros of a class of generalised Dirichlet seriesXVIII, Hardy-Ramanujan J., vol. 20 (1997), 12-28.

[6] K. RAMACHANDRA, On the zeros of a class of generalised Dirichlet series-V, J. Reine a. Angew. Math., 303/304 (1978), 295-313.

[7] K. RAMACHANDRA, Some remarks on a theorem of Montgomery and Vaughan, J. Number Theory, 11 (1979), 465-471.

[8] E.C. TITCHMARSH, The Theory of the Riemann zeta-function (Revised and edited by D.R. HEATH-BROWN), Clarendon Press, Oxford (1986). 


\section{ADDRESS OF THE AUTHOR}

K. RAMACHANDRA

SENIOR PROFESSOR

SCHOOL OF MATHEMATICS

TATA INSTITUTE OF FUNDAMENTAL RESEARCH

HOMI BHABHA ROAD

BOMBAY 400 005, INDIA

e-mail : KRAM@TIFRVAX.TIFR.RES.IN

MANUSCRIPT COMPLETED ON 22nd OCTOBER 1995. 